\title{
Acoustic Simulation of Coaxial Hot Air Jets Using Cold Helium-Air Mixture Jets
}

\author{
Dimitri Papamoschou* \\ University of California, Irvine, Irvine, California 92697-3975
}

DOI: $10.2514 / 1.21776$

\begin{abstract}
This work examines the ability of small-scale helium-air mixture coaxial jets to simulate the acoustics of largescale hot air jets representing the exhaust of separate-flow turbofan engines. Experiments employed a one-eighthscale model of a separate-flow nozzle used in hot tests at NASA John H. Glenn Research Center. Comparisons were conducted for two set points using the following methods: matching velocity and density, and matching velocity and Mach number. For both methods, the helium-air data compare well with the hot data in all measures of noise: spectral shapes, spectral levels, and overall sound pressure levels. The method of matching velocity and Mach number gives slightly better agreement in the spectral shapes at angles close to the jet axis and in the overall sound pressure levels. The overall agreement between the hot air and helium-air mixture data is within $1.2 \mathrm{~dB}$.
\end{abstract}

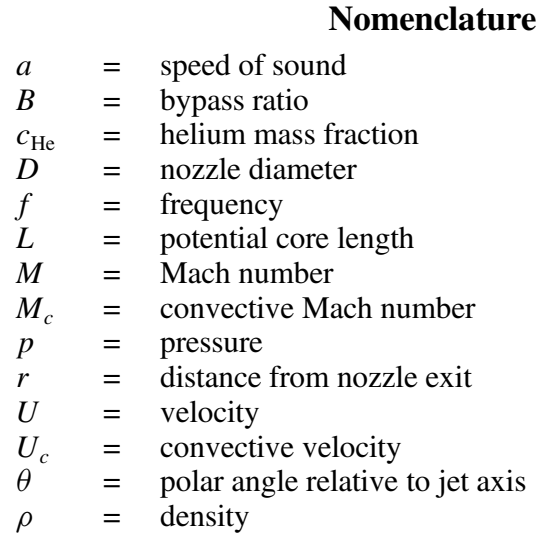

Subscripts

$f \quad=$ fan

hot $=$ heated air

$\operatorname{mix}=$ cold helium-air mixture

$p \quad=$ primary (core) stream

$s \quad=\quad$ secondary (bypass) stream

$0 \quad=$ total (stagnation)

$\infty=$ ambient

\section{Introduction}

$\mathbf{J}$ ET aeroacoustics remains one of the most challenging fields of continuum mechanics. In the last decade substantial progress has been made in the understanding and modeling of jet noise. Advanced numerical methods are showing promise in computing from first principles the sound emitted by complex jets [1], but it will take many years until computers are powerful enough to resolve accurately the sound spectrum relevant to community noise. For the foreseeable future, experiments offer the only way to obtain reliable acoustic data. Given the wealth of variables influencing sound emission

Presented as Paper 0208 at the AIAA 43d Aerospace Sciences Meeting, Reno, NV, 10-13 January 2005; received 13 December 2005; revision received 14 May 2006; accepted for publication 26 June 2006. Copyright () 2006 by D. Papamoschou. Published by the American Institute of Aeronautics and Astronautics, Inc., with permission. Copies of this paper may be made for personal or internal use, on condition that the copier pay the $\$ 10.00$ per-copy fee to the Copyright Clearance Center, Inc., 222 Rosewood Drive, Danvers, MA 01923; include the code 0748-4658/07 \$10.00 in correspondence with the CCC.

*Professor, Department of Mechanical and Aerospace Engineering. Associate Fellow AIAA. (velocity, Mach number, density, bypass ratio, nozzle shape, etc.), experiments need to cover a large parameter space to produce data meaningful for aircraft noise prediction and control. This can become a very expensive process.

Jet aeroacoustic facilities in the United States employing nozzles with realistic geometry and realistic flow conditions are about $1 / 10$ the scale of the actual engine. They exist at NASA centers and industrial labs. These facilities have been instrumental in the advancement of knowledge on jet noise and the development of novel concepts for noise suppression [2]. There is no substitute for their capability and scientific value. However, their operation is very expensive, and investigations can be lengthy.

University facilities tend to be an order of magnitude smaller, about $1 / 100$ to $1 / 50$ scale. The majority of university experiments have used simple nozzles to investigate single-stream jets composed of cold air. Few rigs are equipped to run hot, fewer are dual stream, and a very small fraction use nozzles representative of those of turbofan engines. It should be realized that a heated jet experiment in a university setting is an expensive proposition fraught with safety and environmental concerns. Although it has proven doable, one sacrifices the flexibility, low cost, and low risk of a cold experiment.

A method to simulate the conditions of a heated air jet, while still running "cold," is to replace the air with a gas, or a gas mixture, with lower molecular weight. Helium is a prime candidate because of its very low molecular weight and because it is nontoxic and noncombustible. For a small-scale facility, the use of helium-air mixtures offers a cheaper, simpler, and intrinsically safer alternative to heating the air. The use of helium-air mixture jets for the study of jet aeroacoustics was pioneered by Chan and Westley [3] and subsequently was used by Kinzie and McLaughlin [4] and several other groups, including the aeroacoustics program at University of California, Irvine (UCI) [5]. In comparing single-stream jets composed of helium-air mixtures with hot air jets, Kinzie and McLaughlin demonstrated reasonable agreement in the acoustics and mean flow characteristics.

A question that invariably arises is can very-small-scale heliumair mixture jets duplicate the acoustics of realistic full-scale heated jets? With regard to turbofan engines, realism involves the exhaust conditions and the shape of the nozzle. An additional element of reality is that, today, all commercial engines have dual-stream exhausts. Even when the core and bypass streams are mixed internally, the exit flow is not uniform enough to be characterized as single stream. The present study addresses the question by surveying the acoustics of very-small-scale helium-air mixture coaxial jets issuing from a nozzle with realistic geometry and comparing them with the acoustics of equivalent large-scale heated jets tested at the Nozzle Acoustic Test Rig of NASA John H. Glenn Research Center. The paper gives a short introduction on the use of helium-air 
mixtures, describes the experimental facilities, and compares the data from the two experiments.

\section{Helium-Air Mixtures}

A comprehensive treatment on the use of helium-air mixtures for jet aeroacoustics can be found in Doty and McLaughlin [6]. Presented here is a brief overview of the ability of helium-air mixtures to capture the acoustics and fluid mechanics of heated air jets. There are three basic fluid mechanical parameters that impact jet mixing noise: velocity, density, and Mach number. Other variables, e.g., temperature, can be expressed in terms of those three parameters. Lighthill's acoustic analogy has shown that sound intensity depends strongly on jet exit velocity. For low-speed jets, dimensional analysis of Lighthill's equation shows that the acoustic intensity scales as $U^{8}$, a trend corroborated by experiments in cold jets [7]. For hot jets, the power-law exponent ranges from 5.5 to 9.8 [8], depending on the direction of radiation and the temperature ratio, and so the acoustic intensity is still a very strong function of velocity. It is therefore the premise of this study that the velocity $U$ must be matched. Assuming that the jet exit pressure is matched with the ambient pressure $p_{\infty}$, the velocity of the hot air jet is

$$
U_{\text {hot }}=M_{\text {hot }} a_{\text {hot }}=M_{\text {hot }} \sqrt{\gamma_{\text {hot }} p_{\infty} / \rho_{\text {hot }}}
$$

and the velocity of the helium-air mixture jet is

$$
U_{\text {mix }}=M_{\text {mix }} a_{\text {mix }}=M_{\text {mix }} \sqrt{\gamma_{\text {mix }} p_{\infty} / \rho_{\text {mix }}}
$$

To match the above velocities, we must have

$$
M_{\text {mix }} \sqrt{\gamma_{\text {mix }} / \rho_{\text {mix }}}=M_{\text {hot }} \sqrt{\gamma_{\text {hot }} / \rho_{\text {hot }}}
$$

For $\gamma_{\text {mix }}=\gamma_{\text {hot }}$ we can match exactly both the density and the Mach number. However, helium is a monatomic gas with specific heat ratio $\gamma=5 / 3$, different from the diatomic value $\gamma=7 / 5=1.4$ for air. A helium-air mixture therefore has $7 / 5<\gamma_{\text {mix }}<5 / 3$. This means that we can match exactly only one of the two parameters (density or Mach number); the remaining parameter will be matched only approximately, within a few percent. In this investigation we compare two approaches: a) matching velocity and density and b) matching velocity and Mach number. Using the velocity matching criterion of Eq. (3), the first approach yields

$$
\rho_{\text {hot }}=\rho_{\text {mix }} \rightarrow \frac{M_{\text {mix }}}{M_{\text {hot }}}=\sqrt{\frac{\gamma_{\text {hot }}}{\gamma_{\text {mix }}}}
$$

and the second approach gives

$$
M_{\mathrm{hot}}=M_{\mathrm{mix}} \rightarrow \frac{\rho_{\mathrm{mix}}}{\rho_{\mathrm{hot}}}=\frac{\gamma_{\mathrm{mix}}}{\gamma_{\mathrm{hot}}}
$$

Because $\gamma_{\text {mix }}>\gamma_{\text {hot }}$, matching the density results in $M_{\text {mix }}<M_{\text {hot }}$. Matching the Mach number gives $\rho_{\text {mix }}>\rho_{\text {hot }}$.

We note one additional difference that affects the experimental procedure but not the fluid mechanics. Assuming perfectly expanded flow, the Mach number is controlled by the nozzle pressure ratio (NPR) $\mathrm{NPR}=p_{0} / p_{\infty}$ via the isentropic relation

$$
M=\sqrt{\frac{2}{\gamma-1}\left[\mathrm{NPR}^{(\gamma-1) / \gamma}-1\right]}
$$

Because of the differences in $\gamma$, at equal $M$ the NPR of the hot air jet is different from the NPR of the cold helium-air mixture jet. The reader is referred to Doty and McLaughlin [6] for further details on the properties and implementation of helium-air mixtures.

\section{Experimental Facilities}

The nozzle of the present experiments is a scaled-down version of the baseline separate-flow nozzle used in heated air tests at NASA John H. Glenn Research Center (GRC). The GRC facility and acoustic data referred to in this paper are covered in Janardan et al. [9]. The baseline nozzle used in the GRC tests is referred to as the $3 \mathrm{BB}$ nozzle $[2,9]$ and so we shall use the same name here. The radial coordinates of the 3BB nozzle were obtained from GRC and were scaled down so that the nozzle mass flow rate would fit the flow capacity of our lab. Stereolithography files were generated, and the nozzle components were rapid prototyped from plastic (epoxy resin) material. Because this material becomes fragile for very small thickness, the relative thickness of the nozzle at the trailing edge is larger than that of the nozzle used in the hot tests. Figure 1 shows the combined stereolithography file of the three elements of the nozzle: fan nozzle, core nozzle, and plug. Figure 2 plots the coordinates of the nozzles used in the hot tests [9] and in the present investigation. The fan exit diameters $D_{f}$ of the nozzles used in the hot and cold tests are 31.0 and $246.1 \mathrm{~mm}$, respectively, giving a scale factor of 7.94 .

The scaled-down 3BB nozzle was tested in the jet aeroacoustics facility depicted in Figs. 3 and 4. The facility supplies mixtures of helium and air to the primary (core) and secondary (bypass) nozzles. The helium mass fraction $c_{\mathrm{He}}$ and the total pressure $p_{0}$ of each mixture are determined by the desired exit velocity and Mach number. The nozzle exit area sets the individual mass flow rates of air and helium. Corresponding to the mass flow rate of air is the total pressure of the air flow alone, $p_{0_{\text {air }}}$. The helium mass fraction is set by first running air alone through the nozzle to match $p_{0_{\text {air }}}$ and then

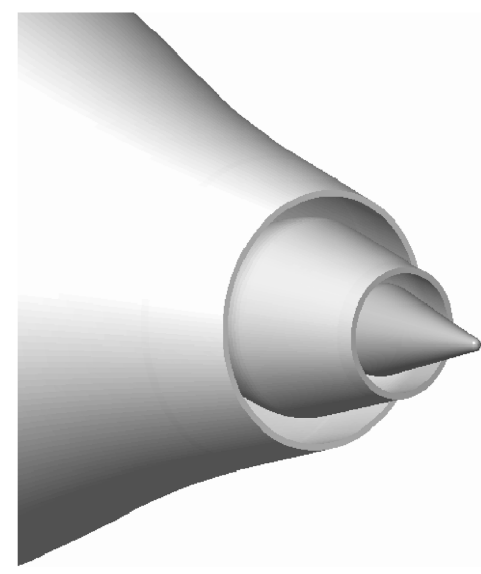

Fig. 1 Stereolithography image of separate-flow nozzle.

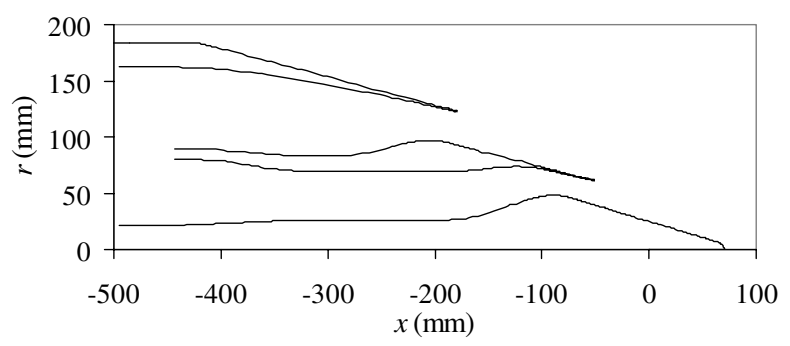

a) Large-scale hot air tests

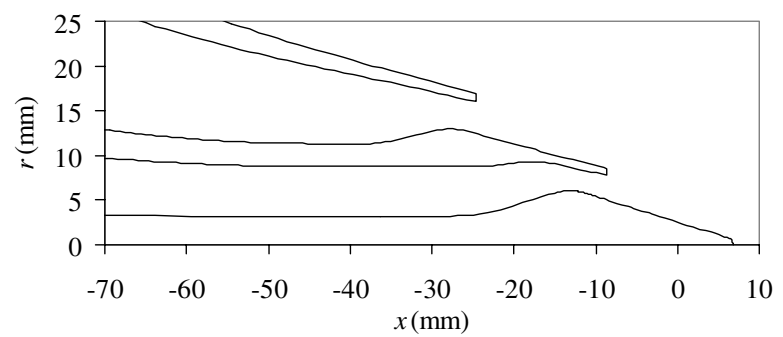

b) Small-scale helium-air mixture

Fig. 2 Radial coordinates of nozzles used in the tests. 


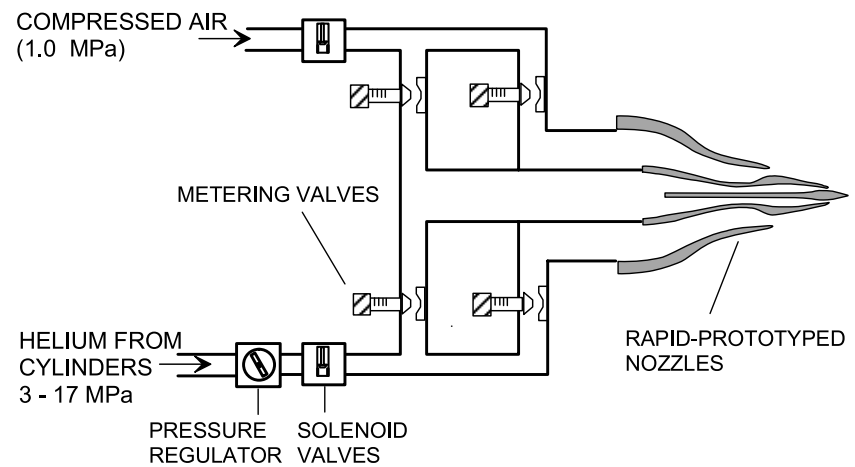

Fig. 3 Dual-stream jet apparatus.

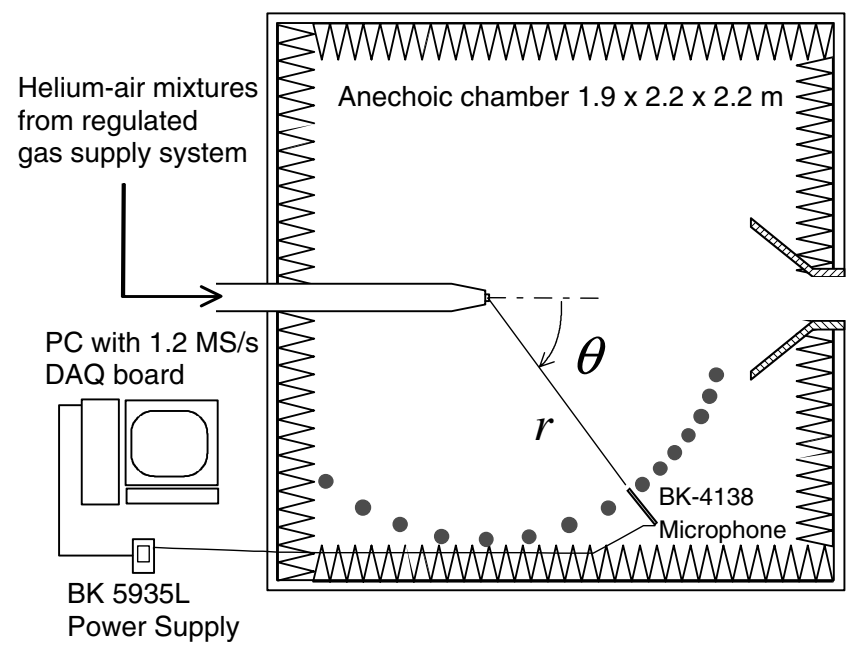

Fig. 4 Jet aeroacoustics facility.

adding helium to match $p_{0}$. This is the same procedure used by Doty and McLaughlin [6].

The experiments attempted to reproduce the conditions of two hot static tests with fully mixed equivalent jet velocities $U_{\text {fmeq }}=354$ and $366 \mathrm{~m} / \mathrm{s}$. The matching methods were the two discussed earlier, i.e., matching velocity and density, and matching velocity and Mach number. For each set point and matching method, the total pressures were held to within $0.5 \%$ of the target values, resulting in errors of $0.3 \%$ in the velocity and $0.2 \%$ in the Mach number. The Reynolds number of the jet, based on fan exit conditions, was $6 \times 10^{5}$. The corresponding Reynolds number of the hot air jet [9] is estimated at $4.8 \times 10^{6}$.

Noise measurements were conducted inside an anechoic chamber using a 3.2-mm condenser microphone (Brüel \& Kjær 4138) with a frequency response of $140 \mathrm{kHz}$. The microphone was mounted on a pivot arm and traced a circular arc centered at the jet exit with radius $r=965 \mathrm{~mm}$, or $r / D_{f}=31.1$. The polar angle $\theta$ ranged from 25 to $130 \mathrm{deg}$ relative to the jet axis. The microphone was sampled at $400 \mathrm{kHz}$ by a fast analog-to-digital board (National Instruments PCI$6070 \mathrm{E}$ ) installed in a Pentium 4 computer. Each recording consisted of 54,280 samples (135 ms), corresponding to the passage of about 10,000 eddies the size of the inner-jet diameter. The signal was highpass filtered at $500 \mathrm{~Hz}$ by a Butterworth filter to remove spurious lowfrequency noise. The narrowband power spectrum of the microphone voltage was computed using a 2048-point fast fourier transform, which provided a spectral resolution of $195 \mathrm{~Hz}$. Using the microphone's sensitivity of $1 \mathrm{mV} / \mathrm{Pa}$ and accounting for the amplifier gain setting, the voltage power spectrum was converted to the power spectrum of $p^{\prime} / p_{\text {ref }}$, where $p^{\prime}$ is the measured pressure fluctuation and $p_{\text {ref }}=20 \mu \mathrm{Pa}$ is the commonly used reference pressure. Converted to units of decibels, the resulting spectrum is raw spectrum of the sound pressure level $(\operatorname{SPL}), \operatorname{SPL}_{\text {raw }}(f)$. This spectrum must undergo several corrections before it becomes accurate. The corrected (lossless) sound pressure level spectrum is given by

$$
\operatorname{SPL}(f)=\operatorname{SPL}_{\text {raw }}(f)-C_{\mathrm{fr}}(f)-C_{\mathrm{ff}}(f)+\alpha(f) r
$$

where $C_{\mathrm{fr}}$ and $C_{\mathrm{ff}}$ are the corrections for the actuator response and free-field response, respectively, and are based on data provided by the manufacturer of the microphone. The atmospheric absorption coefficient $\alpha(\mathrm{dB} / \mathrm{m})$ is computed using the formulas proposed by Bass et al. [10] for the measured values of relative humidity and temperature of the ambient air. Repetition of the same experiment under varying temperature and relative-humidity conditions (typically from 20 to $50 \%$ ) yielded spectra that differed by at most $0.5 \mathrm{~dB}$. The overall sound pressure level (OASPL) was obtained by integrating the lossless spectrum:

$$
\text { OASPL }=10 \log _{10} \int_{0}^{f_{\text {upper }}} 10^{0.1 \operatorname{SPL}(f)} \mathrm{d} f
$$

where the upper limit is the highest frequency that can be resolved, in this case $140 \mathrm{kHz}$.

\section{Comparison Procedure}

The spectra of the large-scale hot jets are published as lossless 1/3octave spectra corresponding to the actual frequencies measured in the hot jet facility [9]. The spectra are referenced to a 305-mm arc centered at the nozzle exit, that is, $r / D_{f}=1.24$. The acoustic data from the helium-air mixture experiments were scaled to the conditions of the hot air tests using the following procedure:

1) The measured frequencies of the helium-air mixture jets were divided by the scale factor 7.94 . The highest resolvable frequency was $140 \mathrm{kHz} / 7.94=17.6 \mathrm{kHz}$.

2) The scaled-up narrowband helium-air mixture spectra were converted into $1 / 3$-octave spectra.

3) The helium-air mixture 1/3-octave spectra were increased incrementally by the distance factor

$$
20 \log _{10}\left[\frac{\left(r / D_{f}\right)_{\text {mix }}}{\left(r / D_{f}\right)_{\text {hot }}}\right]=20 \log _{10}(31.1 / 1.24)=28.0 \mathrm{~dB}
$$

to become directly comparable with the corresponding spectra of the large-scale hot jets.

This procedure reflects the scaling law for the power spectral density proposed by Tam [11], except that the Strouhal scaling is not applicable here because the power in each frequency bin of the $1 / 3$ octave spectrum represents an integrated quantity.

\section{Results and Discussion}

We compare the spectra of the actual (heated air) and simulated (helium-air mixture) jets for two set points $\left(U_{\text {fmeq }}=354 \mathrm{~m} / \mathrm{s}\right.$ and

Table 1 Exit conditions for $U_{\mathrm{fmeq}}=354 \mathrm{~m} / \mathrm{s}$

\begin{tabular}{lccc}
\hline \hline Quantity & Hot & He-air & He-air \\
\hline $\mathrm{NPR}_{p}$ & 1.688 & 1.690 & 1.765 \\
$M_{p}$ & 0.898 & 0.857 & 0.898 \\
$U_{p}, \mathrm{~m} / \mathrm{s}$ & 484 & 485 & 485 \\
$\rho_{p} / \rho_{a}$ & 0.408 & 0.409 & 0.445 \\
$c_{\mathrm{He}, p}$ & $\overline{1.400}$ & 0.305 & 0.281 \\
$\gamma_{p}$ & 1.575 & 1.568 \\
$\mathrm{NPR}_{s}$ & 1.842 & 1.826 & 1.850 \\
$M_{s}$ & 0.976 & 0.962 & 0.975 \\
$U_{s}, \mathrm{~m} / \mathrm{s}$ & 328 & 327 & 328 \\
$\rho_{s} / \rho_{a}$ & 1.044 & 1.043 & 1.061 \\
$c_{\mathrm{He}, s}$ & & 0.027 & 0.023 \\
$\gamma_{s}$ & 1.400 & 1.429 & 1.420 \\
$B$ & 4.98 & 5.15 & 4.88 \\
\hline \hline
\end{tabular}

${ }^{a}$ Matching velocity and density. ${ }^{\mathrm{b}}$ Matching velocity and Mach number. 
Table 2 Exit conditions for $U_{\mathrm{fmeq}}=366 \mathrm{~m} / \mathrm{s}$

\begin{tabular}{lccr}
\hline \hline Quantity & Hot & He-air & He-air \\
\hline $\mathrm{NPR}_{p}$ & 1.780 & 1.884 \\
$M_{p}$ & 1.793 & 0.898 & 0.952 \\
$U_{p}, \mathrm{~m} / \mathrm{s}$ & 0.953 & 514 & 515 \\
$\rho_{p} / \rho_{a}$ & 515 & 0.408 & 0.452 \\
$c_{\mathrm{He}, p}$ & 0.403 & 0.321 & 0.291 \\
$\gamma_{p}$ & \multirow{2}{*}{1.400} & 1.581 & 1.571 \\
$\mathrm{NPR}_{s}$ & 1.898 & 1.890 & 1.905 \\
$M_{s}$ & 1.002 & 0.990 & 1.002 \\
$U_{s}, \mathrm{~m} / \mathrm{s}$ & 336 & 336 & 336 \\
$\rho_{s} / \rho_{a}$ & 1.051 & 1.048 & 1.072 \\
$c_{\mathrm{He}, s}$ & & 0.028 & 0.025 \\
$\gamma_{s}$ & 1.40 & 1.430 & 1.427 \\
$B$ & 4.85 & 5.14 & 4.74 \\
\hline \hline
\end{tabular}

${ }^{\mathrm{a}}$ Matching velocity and density. ${ }^{\mathrm{b}}$ Matching velocity and Mach number.

$366 \mathrm{~m} / \mathrm{s}$ ) using the two methods discussed in Sec. II: matching and velocity and density, and matching velocity and Mach number. Table 1 shows the hot air conditions of the first set point and the corresponding helium-air mixture (He-air) conditions using the aforementioned matching methods. Table 2 shows the analogous information for the second set point. The He-air conditions listed in the tables represent average conditions over the coverage of all the polar angles. Departures from the target values are due to small errors in setting the total pressures, discussed in Sec. III.
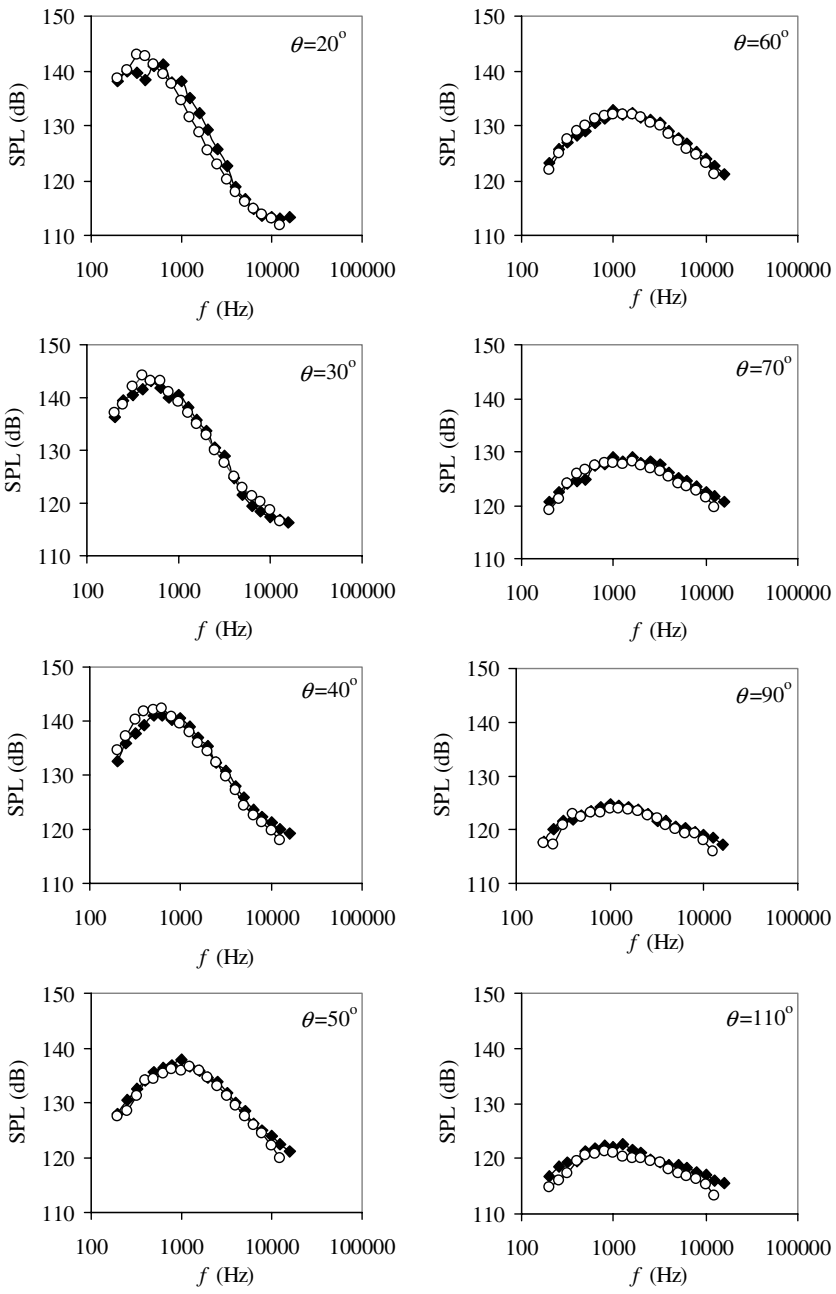

Fig. 5 Spectral comparisons at various polar angles for $U_{\text {fmeq }}=$ $354 \mathrm{~m} / \mathrm{s}$ using the method of matching velocity and density. Solid symbols: hot air jet [9]; open symbols: helium-air mixture jet.
First we examine the method of comparing at equal velocity and density. Spectra at selected polar angles are shown in Figs. 5 and 6 for set points $U_{\text {fmeq }}=354$ and $366 \mathrm{~m} / \mathrm{s}$, respectively. For both set points, the He-air spectra agree well with the hot spectra, except at very small angles $(\theta=20-30 \mathrm{deg})$, where there is a small underprediction of the spectrum at low-to-medium frequencies. Next we compare the actual and simulated jets at equal velocity and Mach number. Spectra at selected polar angles are shown in Figs. 7 and 8 for set points $U_{\text {fmeq }}=354$ and $366 \mathrm{~m} / \mathrm{s}$, respectively. We note very good agreement for all the polar angles.

Figure 9 plots the OASPL directivities of the hot and He-air data using the method of matching velocity and density. Although the overall agreement is very good, the $\mathrm{He}$-air data slightly underpredict the OASPL at the larger polar angles. Comparison at the same velocity and Mach number, Fig. 10, produces even better agreement, and the underprediction at the large angles becomes very small. The agreement between the $\mathrm{He}$-air and hot data can be quantified in terms of the root mean square deviation

$$
\Delta \text { OASPL }=\sqrt{\frac{1}{N} \sum_{k=1}^{N}\left(\mathrm{OASPL}_{\text {mix }, k}-\mathrm{OASPL}_{\mathrm{hot}, k}\right)^{2}}
$$

where the summation is over the 14 polar angles measured in the UCI experiment. For the method of matching velocity and density, the deviation is 1.3 and $1.4 \mathrm{~dB}$ for $U_{\text {fmeq }}=354$ and $366 \mathrm{~m} / \mathrm{s}$, respectively. When matching velocity and Mach number, the corresponding deviations are 1.2 and $1.0 \mathrm{~dB}$.
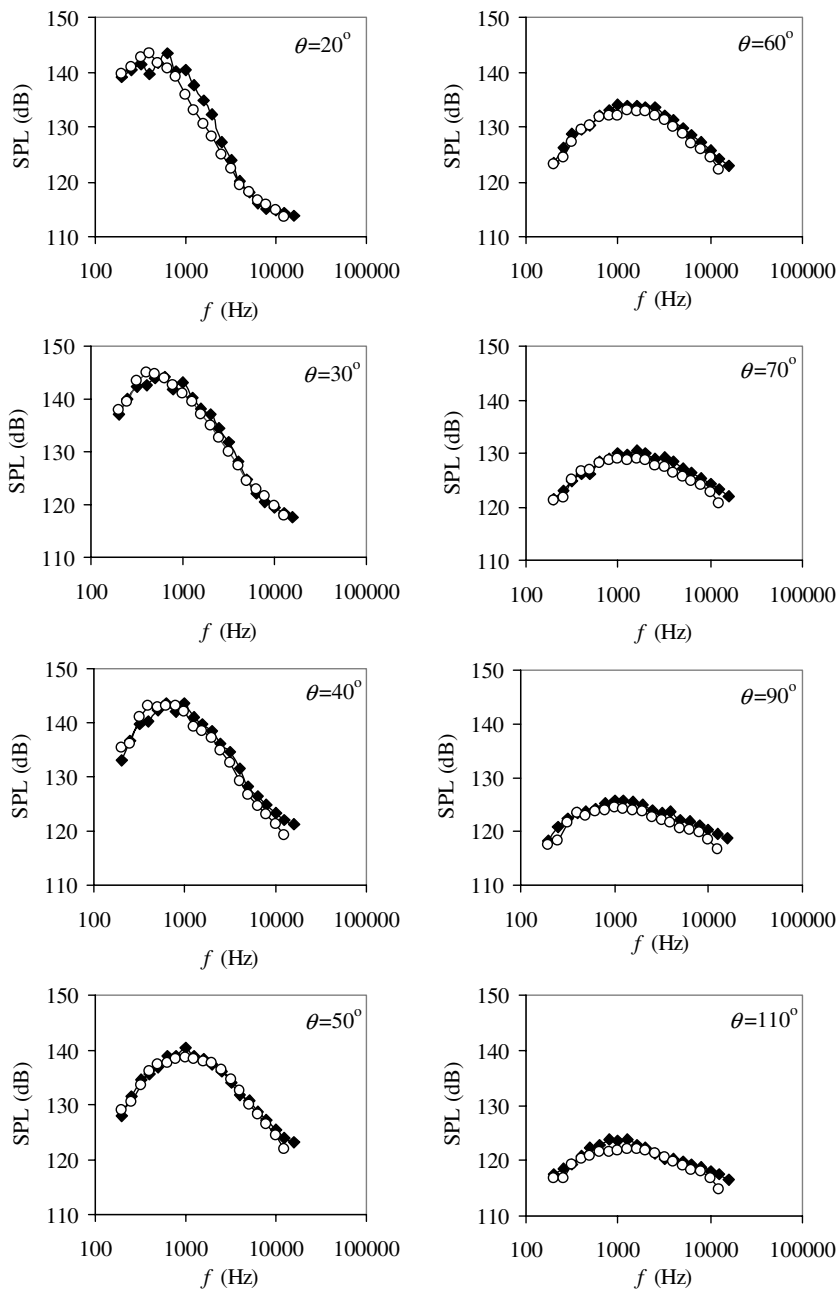

Fig. 6 Spectral comparisons at various polar angles for $U_{\mathrm{fmeq}}=$ $366 \mathrm{~m} / \mathrm{s}$ using the method of matching velocity and density. Solid symbols: hot air jet [9]; open symbols: helium-air mixture jet. 

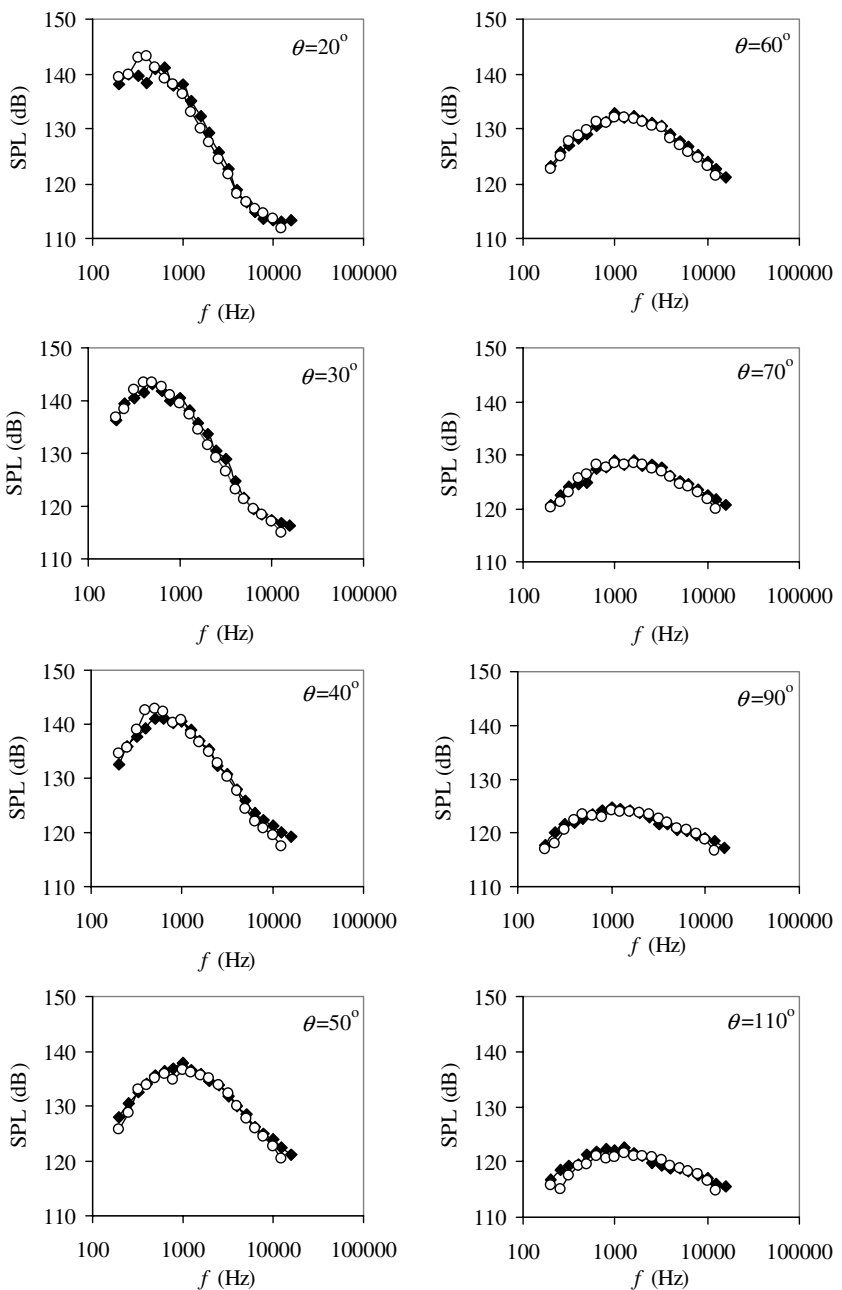

Fig. 7 Spectral comparisons at various polar angles for $U_{\text {fmeq }}=$ $354 \mathrm{~m} / \mathrm{s}$ using the method of matching velocity and Mach number. Solid symbols: hot air jet [9]; open symbols: helium-air mixture jet.

Even though the differences between the results of the two matching methods are small, it is worth analyzing some basic fluid mechanics to gain additional insight as to which method is preferable. It is widely agreed that turbulent shear-flow mixing causes two types of noise: sound produced by large-scale eddies and sound generated by fine-scale turbulence [12]. The former is very intense and directed at angles close to the jet axis. The latter is nearly uniform and affects the lateral and upstream directions. Large-scale mixing noise has been successfully modeled by treating the eddies as instability waves. Sound radiation is governed by the convective velocity $U_{c}$ of the instability wave. When $U_{c}$ is supersonic, strong Mach wave radiation is evident in instantaneous photographic realizations of jets. For subsonic $U_{c}$, the growth-decay nature of instability waves creates a spectrum of phase speeds, part of which are supersonic [12]. The resulting Mach wave emission is not as intense or nonlinear as its supersonic counterpart but still constitutes the strongest source of sound.

Direct measurements of $U_{c}$ by Murakami and Papamoschou [13] and by Thurow et al. [14] have shown that $U_{c}$ it is strongly dependent on the convective Mach number $M_{c}$ of the turbulent mixing region. Here we use the "symmetric" definition of $M_{c}$ [13] as an overall measure of shear-layer compressibility. The coaxial jets of the present study have a long primary potential core $L_{p}$ and a short secondary potential core $L_{s}$. For both set points and both matching methods, the mean flow model of Murakami and Papamoschou [15] gives the following estimates: $L_{p} / D_{f}=4.7$ and $L_{s} / D_{f}=1.6$, both lengths being measured from the exit of the primary nozzle $(x=-9 \mathrm{~mm}$ in Fig. $2 \mathrm{~b}$ ). The upshot is that a significant region of the primary potential core is not surrounded by the secondary potential
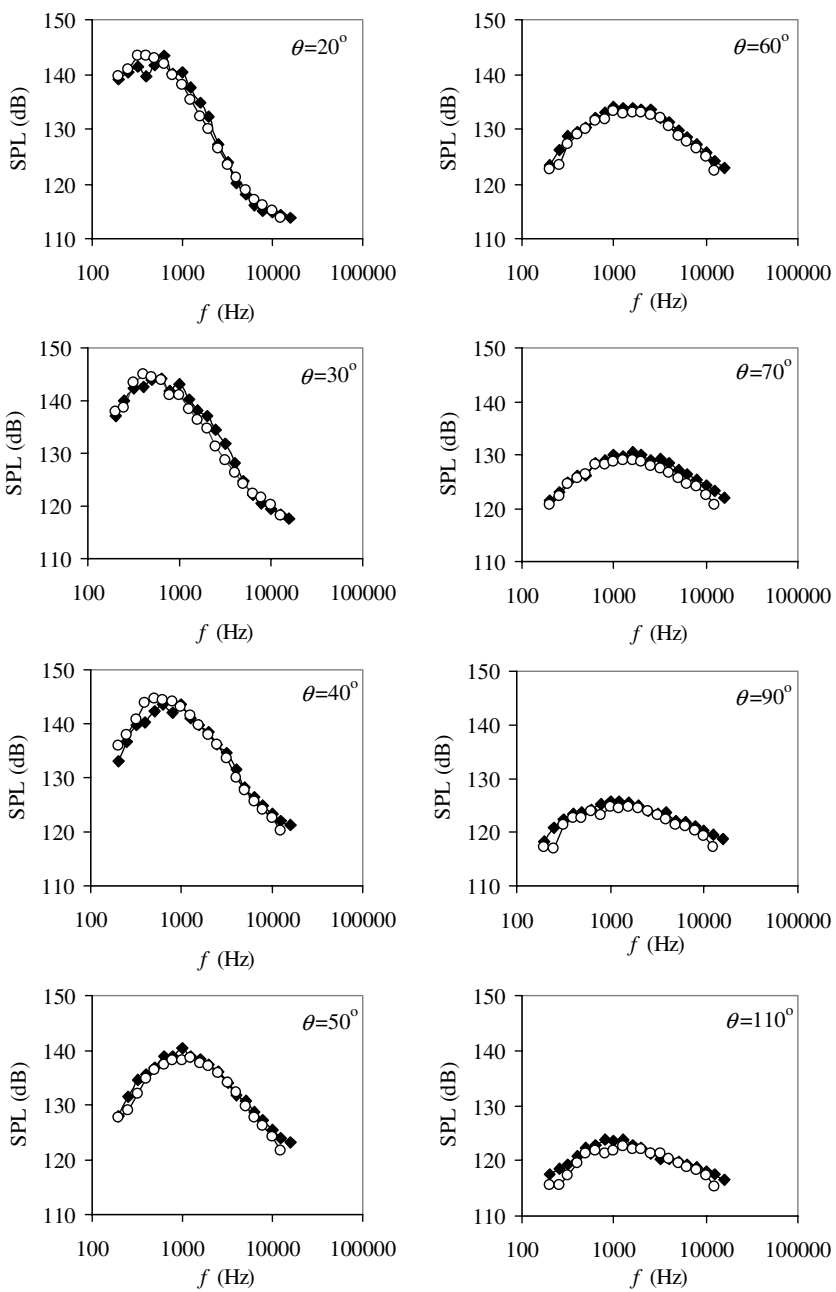

Fig. 8 Spectral comparisons at various polar angles for $U_{\mathrm{fmeq}}=$ $366 \mathrm{~m} / \mathrm{s}$ using the method of matching velocity and Mach number. Solid symbols: hot air jet [9]; open symbols: helium-air mixture jet.

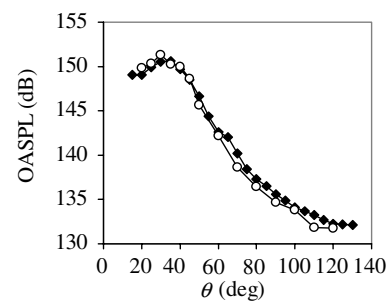

a) $U_{\{\text {fmeq }\}}=345 \mathrm{~m} / \mathrm{s}$

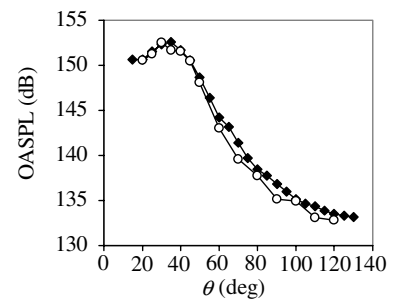

b) $U_{\{\text {fmeq }\}}=366 \mathrm{~m} / \mathrm{s}$
Fig. 9 Comparison of OASPL using the method of matching velocity and density. Solid symbols: hot air jet [9]; open symbols: helium-air mixture jet.

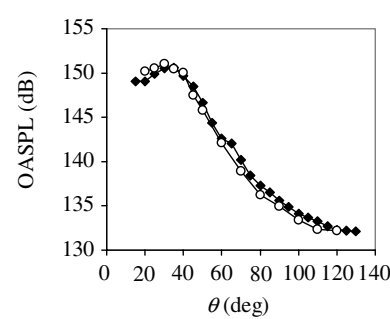

a) $U_{\{\text {fmeq }\}}=354 \mathrm{~m} / \mathrm{s}$

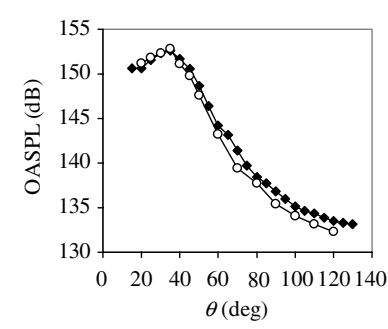

b) $U_{\{\mathrm{fmeq}\}}=366 \mathrm{~m} / \mathrm{s}$
Fig. 10 Comparison of OASPL using the method of matching velocity and Mach number. Solid symbols: hot air jet [9]; open symbols: heliumair mixture jet. 
core; this so-called "intermediate region" can be treated effectively as a single jet with velocity $U_{p}$ [16]. With regard to noise emission, the near-field of the jet encompasses two important regions: the shear layer between the primary potential core and the ambient in the intermediate region, and the shear layer between the secondary potential core and the ambient very near the nozzle exit [16]. For a quiescent ambient, the respective convective Mach numbers are

$$
M_{c_{p, \infty}}=\frac{U_{p}}{a_{p}+a_{\infty}}=\frac{M_{p}}{1+\sqrt{\left(\gamma_{\infty} / \gamma_{p}\right)\left(\rho_{p} / \rho_{\infty}\right)}}
$$

and

$$
M_{c_{s, \infty}}=\frac{U_{s}}{a_{s}+a_{\infty}}=\frac{M_{s}}{1+\sqrt{\left(\gamma_{\infty} / \gamma_{s}\right)\left(\rho_{s} / \rho_{\infty}\right)}}
$$

Let us now examine one of these regions and consider the substitution of hot air by a helium-air mixture. For the exhaust temperatures of modern turbofan engines $\left(\leq 700^{\circ} \mathrm{K}\right)$, vibrational modes have negligible impact on the specific heat ratio [17] and so $\gamma_{\text {hot }}=\gamma_{\infty}=1.4$. Dropping for simplicity the regional subscripts, the convective Mach number of the heated region is

$$
M_{c_{\mathrm{hot}}}=\frac{M_{\mathrm{hot}}}{1+\sqrt{\rho_{\mathrm{hot}} / \rho_{\infty}}}
$$

For the helium-air mixture jet, we have

$$
M_{c_{\text {mix }}}=\frac{M_{\text {mix }}}{1+\sqrt{\left(\gamma_{\infty} / \gamma_{\text {mix }}\right)\left(\rho_{\text {mix }} / \rho_{\infty}\right)}}
$$

Consider the option of matching velocity and density [Eq. (4)]. The relation between $M_{c_{\text {hot }}}$ and $M_{c_{\text {mix }}}$ is

$$
M_{c_{\text {mix }}}=M_{c_{\text {hot }}} \frac{1+\sqrt{\rho_{\text {hot }} / \rho_{\infty}}}{\sqrt{\gamma_{\text {mix }} / \gamma_{\infty}}+\sqrt{\rho_{\text {hot }} / \rho_{\infty}}}
$$

Because $\gamma_{\text {mix }}>\gamma_{\infty}, M_{c_{\text {mix }}}<M_{c_{\text {hot }}}$. On the other hand, when matching velocity and Mach number [Eq. (5)], the speed of sound is also matched resulting in perfect equality of the convective Mach numbers:

$$
M_{c_{\text {mix }}}=M_{c_{\text {hot }}}
$$

This observation is significant because, as noted earlier, the convective velocity is strongly dependent on $M_{c}$. It affects mainly the primary (core) flow where there is noticeable departure of $\gamma$ from the ambient value. The convective speeds for the primary and secondary shear layers were estimated using the empirical model of Murakami and Papamoschou [13]. They are listed together with the convective

Table 3 Estimated convective velocities, $U_{\mathrm{fmeq}}=354 \mathrm{~m} / \mathrm{s}$

\begin{tabular}{llcc}
\hline \hline Quantity & Hot & He-air & He-air \\
\hline$M_{c_{p, \infty}}$ & 0.547 & 0.532 & 0.547 \\
$M_{c_{s, \infty}}$ & 0.483 & 0.479 & 0.483 \\
$U_{c_{p}}, \mathrm{~m} / \mathrm{s}$ & 292 & 279 & 293 \\
$U_{c_{s}}, \mathrm{~m} / \mathrm{s}$ & 214 & 211 & 214 \\
\hline \hline
\end{tabular}

${ }^{\mathrm{a}}$ Matching velocity and density. ${ }^{\mathrm{b}}$ Matching velocity and Mach number.

Table 4 Estimated convective velocities, $U_{\mathrm{fmeq}}=366 \mathrm{~m} / \mathrm{s}$

\begin{tabular}{llcc}
\hline \hline Quantity & Hot & He-air & He-air \\
\hline$M_{c_{p, \infty}}$ & 0.581 & 0.561 & 0.581 \\
$M_{c_{s, \infty}}$ & 0.495 & 0.493 & 0.496 \\
$U_{c_{p}}, \mathrm{~m} / \mathrm{s}$ & 324 & 306 & 324 \\
$U_{c_{s}}, \mathrm{~m} / \mathrm{s}$ & 225 & 224 & 225 \\
\hline \hline
\end{tabular}

${ }^{\mathrm{a}}$ Matching velocity and density. ${ }^{\mathrm{b}}$ Matching velocity and Mach number.
Mach numbers in Tables 3 and 4 for $U_{\text {fmeq }}=354$ and $366 \mathrm{~m} / \mathrm{s}$, respectively. When matching velocity and density, the primary convective speed $U_{c_{p}}$ of the helium-air mixture jet is about $5 \%$ lower than that of the hot jet. When matching velocity and Mach number, there is a near-perfect equality of convective speeds between the actual and simulated jets, a consequence of the equality of the convective Mach numbers. The lower estimated $U_{c_{p}}$ of the densitymatching method may explain the underprediction of the spectral levels at shallow angles (Figs. 5 and 6), a direction influenced by noise from large-scale turbulent structures.

\section{Conclusions}

The acoustics of large-scale heated coaxial air jets issuing from realistic nozzles are successfully simulated by equivalent small-scale cold helium-air mixture jets. The study covers two set points and polar angles ranging from 20 to $130 \mathrm{deg}$ relative to the jet axis. The helium-air mixture jets are compared with hot air jets using two methods: a) matching velocity and density, and b) matching velocity and Mach number. Both methods yield good agreement in the spectral shapes, spectral levels, and overall sound pressure levels, with method b) having a small but consistent advantage. Estimation of convective velocities of large-scale turbulent structure indicates that method b) reproduces these velocities better than method a). This is a possible explanation for its superior acoustic matching.

\section{Acknowledgments}

This work was supported by NASA John H. Glenn Research Center, Grant No. NAG-3-2345 (grant monitors K. Zaman and J. Bridges). J. Bridges is thanked for providing the NASA John $\mathrm{H}$. Glenn Research Center nozzle coordinates and acoustic data. Kimberley Nishi is also thanked for her assistance with nozzle design and testing.

\section{References}

[1] Shur, M. L., Spalart, P. R., and Strelets, M. K., "Noise Prediction of Increasingly Complex Jets, Part I: Methods and Tests," International Journal of Aeroacoustics, Vol. 4, Nos. 3-4, 2005, pp. 213-246.

[2] Saiyed, N. H., Mikkelsen, K. L., and Bridges, J. E., "Acoustics and Thrust of Separate-Flow High-Bypass-Ratio Engines," AIAA Journal, Vol. 41, No. 3, 2003, pp. 372-378.

[3] Chan, Y. Y., and Westley, R., "Directional Acoustic Radiation Generated by Spatial Jet Instability," CASI Transactions, Vol. 6, No. 1, 1973, pp. 36-41.

[4] Kinzie, K. W., and McLaughlin, D. K., "Measurements of Supersonic Helium/Air Mixture Jets," AIAA Journal, Vol. 37, No. 11, 1999, pp. 1363-1369.

[5] Papamoschou, D., and Debiasi, M., "Noise Measurements in Supersonic Jets Treated with the Mach Wave Elimination Method," AIAA Journal, Vol. 37, No. 2, 1999, pp. 154-160.

[6] Doty, M. J., and McLaughlin, D. K., "Acoustic and Mean Flow Measurements of High-Speed, Helium-Air Jets," International Journal of Aeroacoustics, Vol. 2, Nos. 3-4, 2003, pp. 293-333.

[7] Dowling, A., and Ffowcs-Williams, J., Sound and Sources of Sound, Ellis Horwood, London, 1983, pp. 157-163.

[8] Viswanathan, K., "Aeroacoustics of Hot Jets," Journal of Fluid Mechanics, Vol. 516, Oct. 2004, pp. 39-82.

[9] Janardan, B. A., Hoff, G. E., Barter, J. W., Martens, S., Gliebe, P. R., Mengle, V., and Dalton, W. N., "AST Critical Propulsion and Noise Reduction Technologies for Future Commercial Subsonic Engines Separate-Flow Exhaust System Noise Reduction Concept Evaluation," NASA CR 2000-210039, Dec. 2000.

[10] Bass, H. E., Sutherland, L. C., Blackstock, D. T., and Hester, D. M., "Atmospheric Absorption of Sound: Further Developments," Journal of the Acoustical Society of America, Vol. 97, No. 1, 1995, pp. 680-683.

[11] Tam, C. K. W., "Dimensional Analysis of Jet-Noise Data," AIAA Journal, Vol. 44, No. 3, 2006, pp. 512-522.

[12] Tam, C. K. W., “Jet Noise: Since 1952," Theoretical and Computational Fluid Dynamics, Vol. 10, Nos. 1-4, Jan. 1998, pp. 393-405.

[13] Murakami, E., and Papamoschou, D., "Eddy Convection in Supersonic Coaxial Jets," AIAA Journal, Vol. 38, No. 4, 2000, pp. 628-635.

[14] Thurow, B., Hileman, J., Lempert, W., and Samimy, M., "A Technique 
for Real-Time Visualization of Flow Structure in High-Speed Flows," Physics of Fluids, Vol. 14, No. 10, 2002, pp. 3449-3452.

[15] Murakami, E., and Papamoschou, D., "Mean Flow Development in Dual-Stream Compressible Jets," AIAA Journal, Vol. 40, No. 6, 2002, pp. 1131-1138.

[16] Fisher, M. J., Preston, G. A., and Bryce, W. D., "A Modelling of the Noise from Simple Coaxial Jets, Part I: With Unheated Primary Flow,"
Journal of Sound and Vibration, Vol. 209, No. 3, 1998, pp. 385-403.

[17] Liepmann, H. W., and Roshko, A., Elements of Gas Dynamics, 1st ed., Wiley, New York, 1957, p. 37.

T. Shih Associate Editor 